\title{
Performance Analysis of Acquisition Algorithms for Navic
}

\author{
N. Alivelu Manga
}

\begin{abstract}
Indian Regional Navigation Satellite System (IRNSS), is an indigenous navigation system designed and developed by ISRO (Indian Space Research Organization).It is named as NavIC, Navigation with Indian Constellation by Indian Prime Minister. NavIC is designed to have seven satellite constellation that provides reliable position, navigation and timing services over India. The focal modules of NavIC receiver are acquisition, tracking and navigation unit. Among them, acquisition is the data processing unit for detecting satellite signals and their corresponding code phase and carrier frequency. In this paper, various acquisition algorithms like Serial search and Parallel Code Phase search algorithms are analyzed and compared with Cooley-Tukey FFT algorithm and sub-sampled Fast Fourier transform (ssFFT).The results obtained in MATLAB shows that the acquisition computation time for ssFFT based NavIC receiver is faster than parallel FFT acquisition and the Cooley-Tukey FFT IRNSS acquisition algorithm is faster and provides better code phase and carrier frequency values compared to serial search acquisition algorithm.
\end{abstract}

Keywords-IRNSS, NavIC, acquisition, parallel code phase search algorithm, serial search acquisition algorithm, ssFFT, Cooley-Tukey FFT.

\section{INTRODUCTION}

Indian Regional Navigation Satellite System provides position, velocity and time over Indian region. It is developed ISRO and coined as NavIC by Prime Minister of India. The IRNSS project was approved by government of India in June 2006 with a sanction of 1420 Cr (Bhaskaranarayana, A, 2008). The IRNSS constellation consists of 7 satellites of which, three are in GEO stationary and four are in GSO synchronous orbits. The IRNSS provide two services namely Standard Position Service (SPS) and Restricted Service (RS).The main modules of NavIC receiver are acquisition, tracking and navigation unit. Among them, acquisition is the data processing unit for detecting satellite signals and their corresponding code phase and carrier frequency. This paper is planned into six sections. SPS signal generation is discussed in Section II. Section III describes the receiver architecture. Section IV describes the signal conventional acquisition algorithms and ssFFT and Cooley-Tukey acquisition algorithms. Section V shows the simulation results of the explained acquisition algorithms and their performance comparisons and conclusions are presented in Section VI

Revised Manuscript Received on December 30, 2019.

* Correspondence Author

Dr. N.Alivelu Manga, Associate Professor, Department of ECE, Chaitanya Bharathi Institute of Technology (CBIT), Hyderabad, India Email: namanga04@gmail.com

(C) The Authors. Published by Blue Eyes Intelligence Engineering and Sciences Publication (BEIESP). This is an open access article under the CC BY-NC-ND license (http://creativecommons.org/licenses/by-nc-nd/4.0/)

\section{SPS SIGNAL GENERATION}

In the process of generation of L5 signal, P-code and navigation data are together multiplied.

The next step is the application of BPSK (Binary Phase Shift Keying) modulation with the incoming carrier signal. After that, C/A (Coarse/Acquisition) code is multiplied with navigation data, then application of BPSK modulation with 90degrees phase shifted version of incoming carrier signal. Obtained results from the two modulators are added get L5 signal of frequency $1175.45 \mathrm{MHz}$ (Fig. 1). To generate L5 signal of frequency $1176.45 \mathrm{MHz}$, a common frequency signal of frequency, $\mathrm{f}_{\mathrm{o}}=10.23 \mathrm{MHz}$ is used.

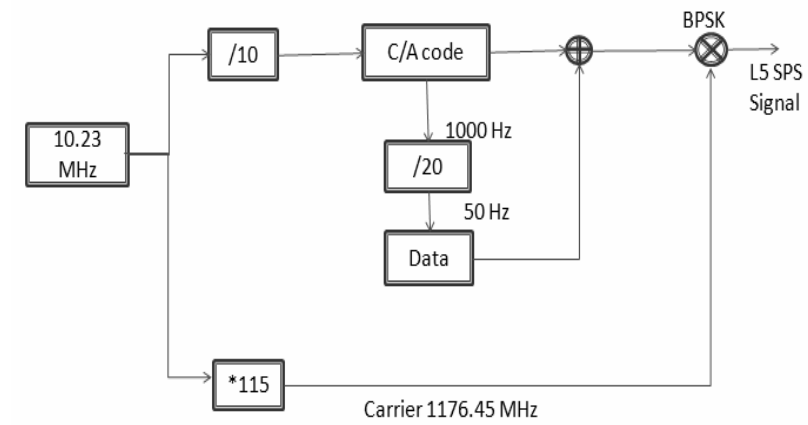

Fig. 1 SPS signal generator Block diagram

The modulo-2 $(\oplus)$ addition gives a signal with values $\{1,0\}$ which is then BPSK modulated with carrier signal to obtain a L5 signal with values $\{-1,1\}$. The chipping rate for $\mathrm{C} / \mathrm{A}$ code is considered as $1.023 \mathrm{MHz}$ and navigation data bit rate is 50 $\mathrm{Hz}$.

A unique C/A code is utilized by each of the satellite for the implementation of CDMA. The C/A codes are generated using pseudorandom noise (PRN) codes, also known as Gold codes. Two Linear Feedback Shift Registers (LFSR), G1 and G2 each of 10 bits, generate maximum length PRN codes having a length of $2^{10}-1$ bits. The length of each generated code is 1023 chips. The code chipping rate used is 1.023 Mcps. The polynomials G1 and G2 used for SPS code generation are defined as given below:

$$
\begin{gathered}
G 1(X)=1+X^{3}+X^{10} \\
G 2(X)=1+X^{2}+X^{3}+X^{6}+X^{8}+X^{9}+X^{10}
\end{gathered}
$$

These polynomials are analogous to the ones used for GPS C/A signal. These polynomials are implemented by using Maximum Length Feedback Shift Registers (MLFSR) each of length 10 bits. The initial state of G2 register provides the chip delay. The all bits of the G1 register are initialized to 1 . Then, the bits of these two registers are exclusive-OR'ed to obtain the final PRN sequence of length 1023 chips. For all 7 satellites, the time period of the PRN sequence is 1 millisecond.

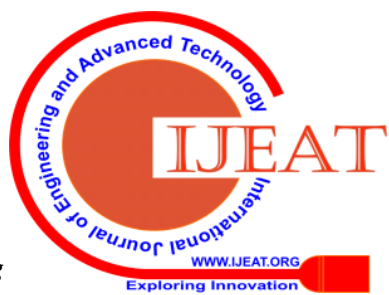




\section{IRNSS RECEIVER ARCHITECTURE}

IRNSS receiver is a system where the Signal In Space (SIS) is processed to determine user's position velocity and time. The functions of various sections of IRNSS receiver (Fig.2) in brief are:

a) Antenna and preamplifier: The L5/S1 frequency band signals are detected by the smart antenna and it converts the wave energy into an electrical current, amplifies the signal strength and passes on the signal receiver electronics. Voltage induced by IRNSS signal in the antenna is sent to the preamplifier.

b)RF/IF Section: The RF signal is mixed with a locally generated sinusoidal signal to down convert IRNSS signal to a lower frequency IF signal. IF signal contains code and data signals from the original RF signal with low carrier frequency.

c) Acquisition : The process of Acquisition detects satellite signal coming from a given IRNSS satellite and provides coarse values of the code phase and the carrier frequency.

d)Tracking: It refines the values produced by acquisition and tracks their changes continuously. It mainly consists of code tracking and carrier tracking loops.

e) NAV unit: The role of an NAV unit block is to compute the IRNSS basic measurements: pseudo range, carrier phase and Doppler shift (or its pseudo range rate version).

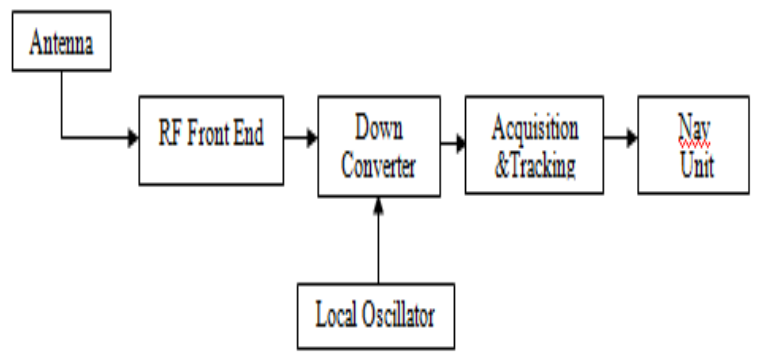

Fig.2 IRNSS Receiver Architecture

\section{ACQUISITION ALGORITHMS}

From the acquisition process, satellite signal is detected and its corresponding coarse values of code phase and carrier frequency are estimated. There are several algorithms for acquisition GNSS/IRNSS signals including Serial search or time domain analysis acquisition algorithm, Parallel code phase search or FFT based algorithm, Parallel frequency code phase search acquisition.

\section{A. Serial Search Acquisition Algorithm}

Serial search acquisition is a simple and is a time domain acquisition algorithm. The process of serial search acquisition is shown in Fig.3. In this locally generated PRN code sequences are multiplied with locally generated replica of the carrier signal. Initially, the received IRNSS signal is multiplied with locally generated PRN sequence. Then, the result is multiplied by a local carrier signal to wipe off the carrier signal from the incoming signal. A in-phase signal I is generated by multiplication with in phase carrier signal replica, and multiplication with 90 degrees phase-shifted carrier signal replica, the quadrature signal $\mathrm{Q}$ is generated. The resulted I and Q signals are combined. This is done based on the length of one PRN code, and are squared and added finally. Code phase value is inferred from the serial search acquisition which gives the correlation between the locally generated signal with that of the incoming signal.

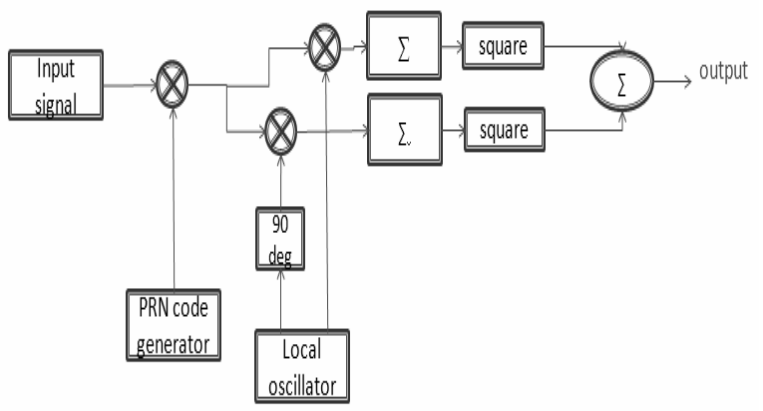

Fig.3 Serial Acquisition

\section{B. Parallel Code Phase Search Acquisition Algorithm}

Parallel Code Phase Search Acquisition Algorithm is Fast Fourier Transform based acquisition algorithm and frequency domain type. In this particular technique, acquisition is done parallel in a code phase dimension to speed up the acquisition process significantly. The received IF signal and the local carrier signal are multiplied together. The in-phase (I) signal is generated by multiplication with in phase signal and quadrature (Q) signal is generated by multiplication with a quad phase-shifted version of the signal,. These signals (I,Q) are combined to result in a complex input to the FFT function. The PRN code, generated for a specific satellite is changed into the frequency domain and complex conjugated. Then, the IF signal and PRN code are perform multiplication in the frequency domain. After that, The result is changed into the time domain by applying an inverse Fourier transform (Fig 4).

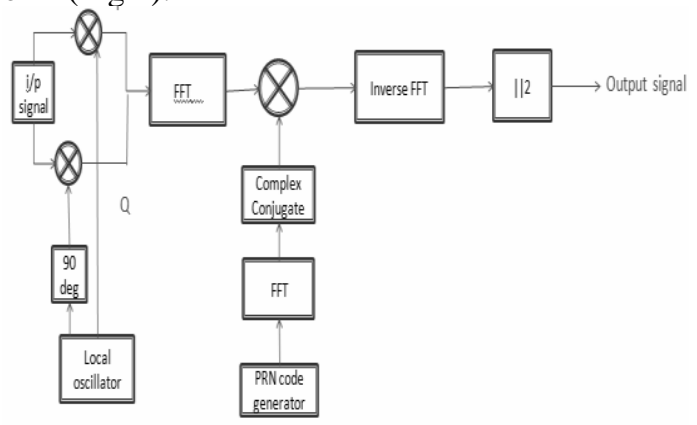

Fig. 4 Parallel Code Phase Search Acquisition Then, the correlation between the input and the PRN code represented by the absolute value of this inverse Fourier transform. The index of the peak presented in the output of correlation, indicates the PRN code phase of the incoming signal.

\section{Sub Sampled Fast Fourier Transform Acquisition}

In this, the signal is down sampled by a factor ' $d$ ', a decimator which is an integer. The IFFT shown in Fig.5 is sparse in the time domain, i.e., there will be only one spike in time domain. Hence, it can be performed in sub-linear time and also require less number of samples as input to compute the sparse IFFT. As a result, there is no need to perform a full length computations on the received signal and one can get all of its frequency samples. Here, the number of frequency samples that are required to perform the sparse inverse FFT need to be computed.

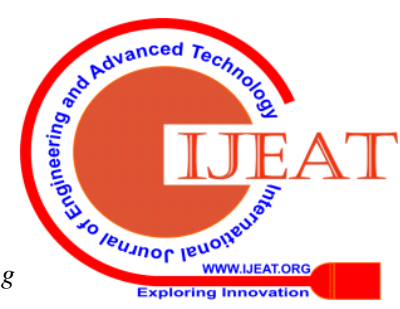




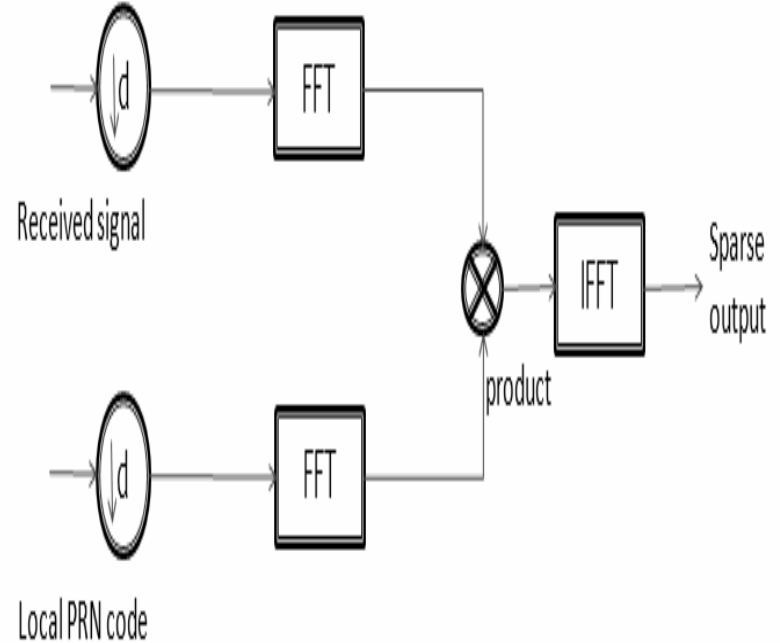

Fig.5 Acquisition using sub sampled Fast Fourier Transform

\section{FFT Acquisition Algorithm using Cooley Tukey FFT}

As shown in Fig.6, the principle of Cooley-Tukey algorithm applied to compute $\mathrm{N}$ point DFT by decomposing into smaller size DFTs, which results in reduction of time of FFT computation.

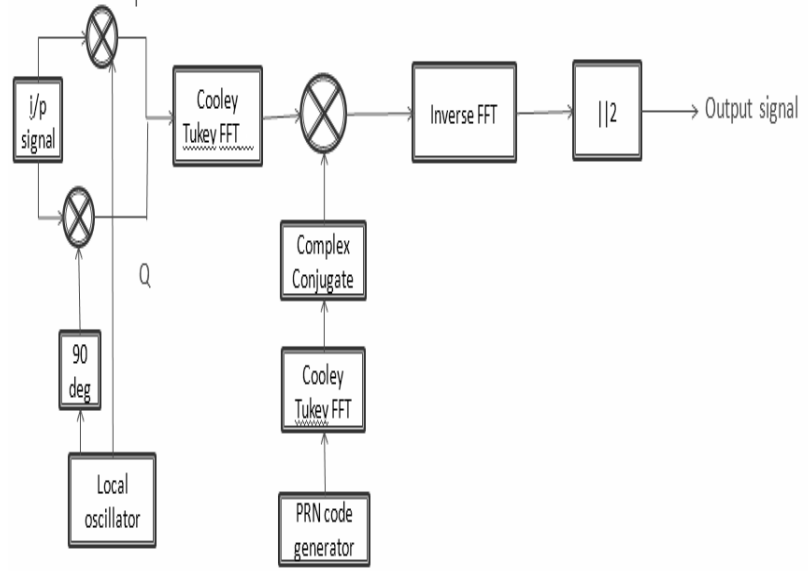

Fig.6 Block diagram of Acquisition based on Cooley Tukey FFT Algorithm

\section{RESULTS AND DISCUSSION}

IRNSS SPS signal is generated for L5 signal and C/A code for different satellites (L5 band) are simulated in MATLAB. The various acquisition algorithms like serial search and parallel code phase search, to find code phase and carrier frequency of received IRNSS signals are simulated in MATLAB. For this, the sampling frequency used is 56 $\mathrm{MHz}$, and carrier frequency is $16.221 \mathrm{MHz}$. Apart from these algorithms, Sub Sampled Fast Fourier Transform Acquisition and FFT Acquisition Algorithm using Cooley Tukey FFT are also analyzed. IRNSS SPS signal for 0.05 milliseconds is shown Fig. 7. In Fig. 8,9,10,11,12,13 and $14 \mathrm{x}$ - axis indicates code phase, $y$ - axis indicates frequency bin and $\mathrm{z}$ - axis indicates magnitude. Doppler shift is calculated using (frequency bin*125-5000).The acquisition results for satellite 4 using serial search method is with Doppler shift $500 \mathrm{~Hz}$ and Code phase 23290 shown in Fig. 8. For satellite 4, the acquisition results using Parallel Code phase search method are presented in Fig 9 with Doppler shift $250 \mathrm{~Hz}$ and Code phase is 853 for down sampled 8184 samples and for
$56000 * 2$ samples Code phase calculated is 23857.Fig 10,11,12 and 13shows Acquisition results for satellite 4 for down sample factor 2,3,4 and 6 respectively. In Fig. 10,Doppler shift is $250 \mathrm{~Hz}$ and Code phase is 426.In Fig. 11, Doppler shift $250 \mathrm{~Hz}$ and Code phase is 284.In Fig. 12 Doppler shift is $250 \mathrm{~Hz}$ and Code phase is 213. In Fig. 13, Doppler shift is $250 \mathrm{~Hz}$ and Code phase is 142.Using Cooley Tukey FFT,8184 samples are divided into 88 and 93 samples. Fig. 14 shows Acquisition results for satellite 4 using Cooley Tukey FFT with Doppler shift is $250 \mathrm{~Hz}$ and code phase is 853.The Table 1 shows the acquisition results for available data using serial search method. The acquisition results using Parallel Code Phase Search Acquisition Algorithm are given in the Table 2. acquisition results of satellite 4 using sub sampled FFT are presented in Table 3. Table 4 shows Acquisition results using Cooley Tukey FFT method. Table 5 shows Comparison of acquisition results for satellite 4 using different acquisition algorithms.

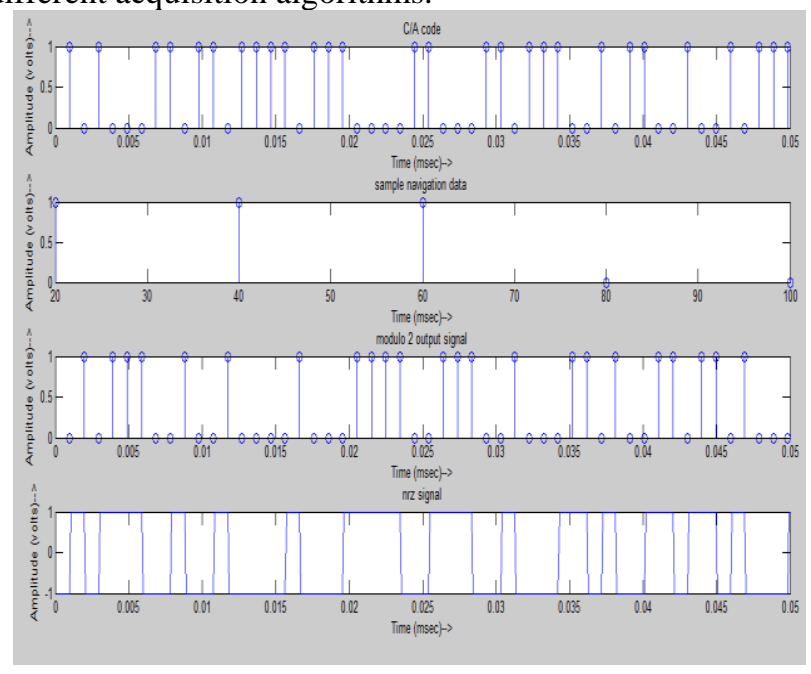

Fig.7 IRNSS SPS generation 


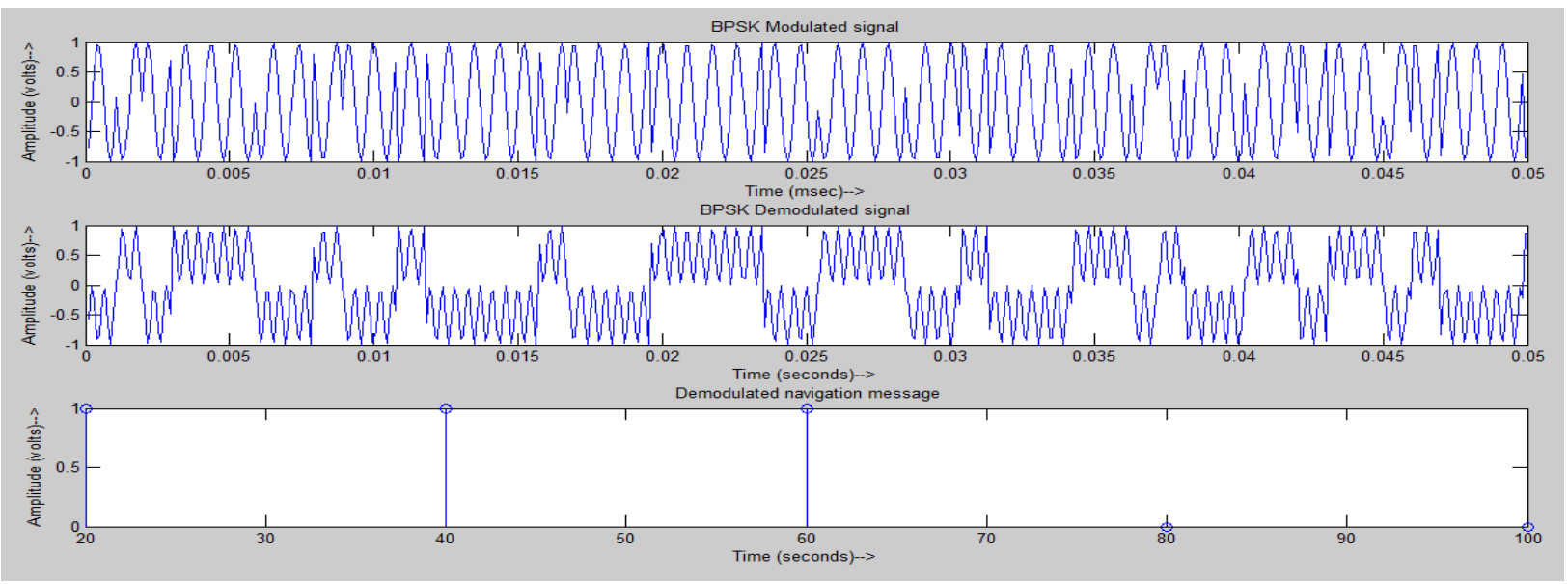

Fig.8 SPS generation for 0.05 milliseconds

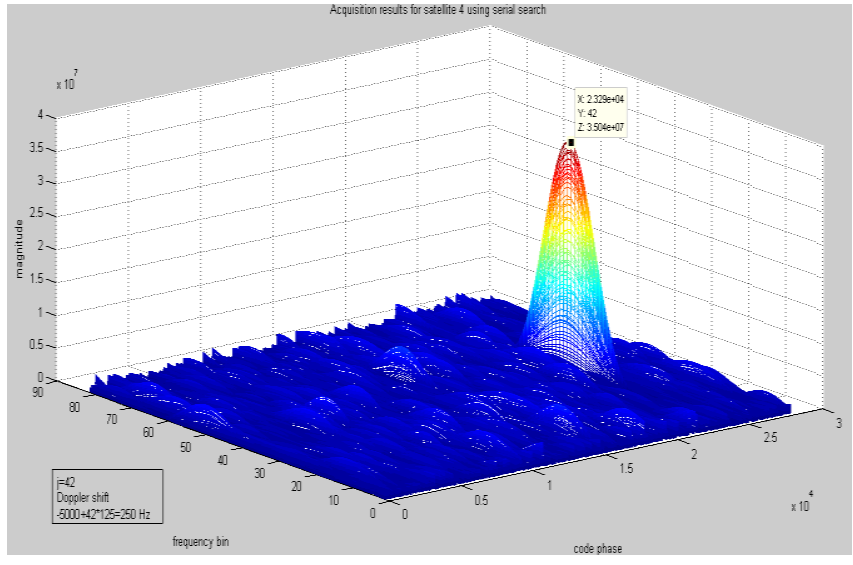

Fig.9 Acquisition results for satellite 4 using serial search method

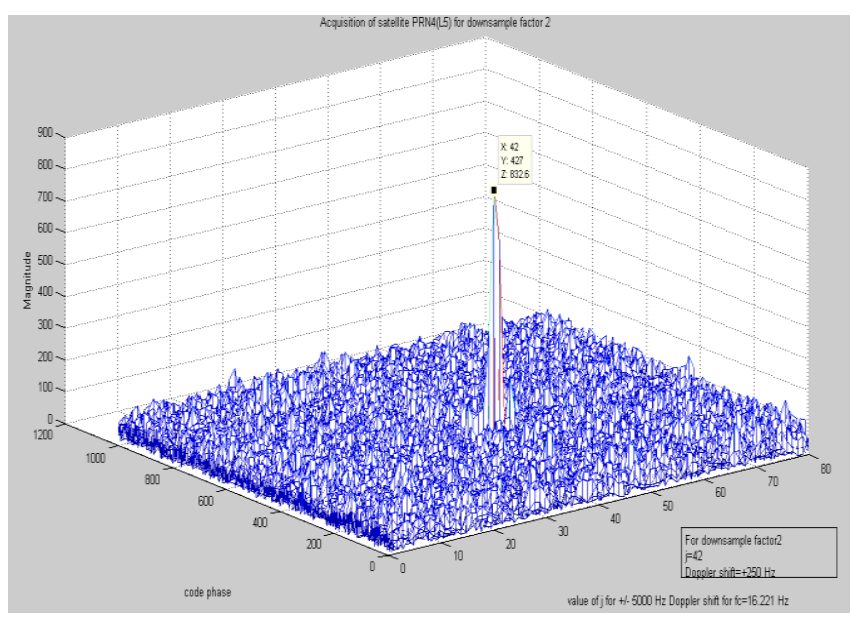

Fig.11 Acquisition results of satellite 4 for down sample factor 2

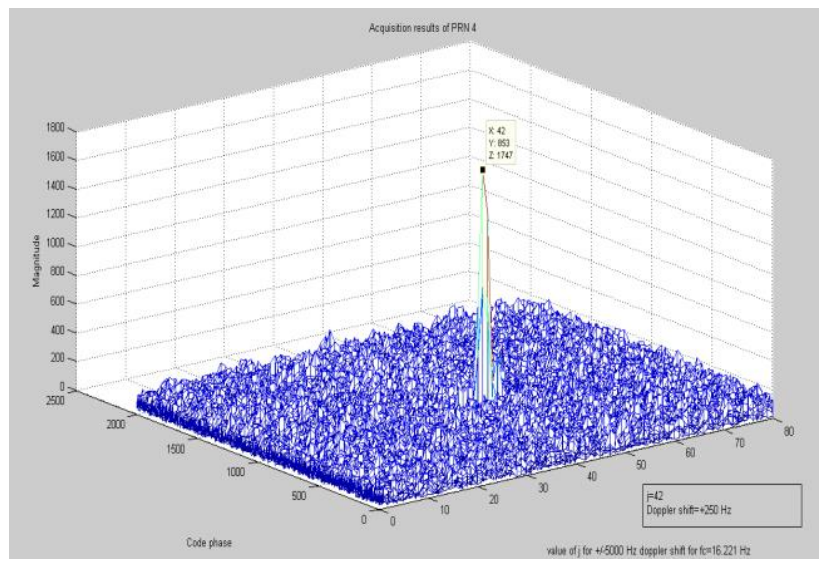

Fig.10 Acquisition results for satellite 4 using parallel code phase method

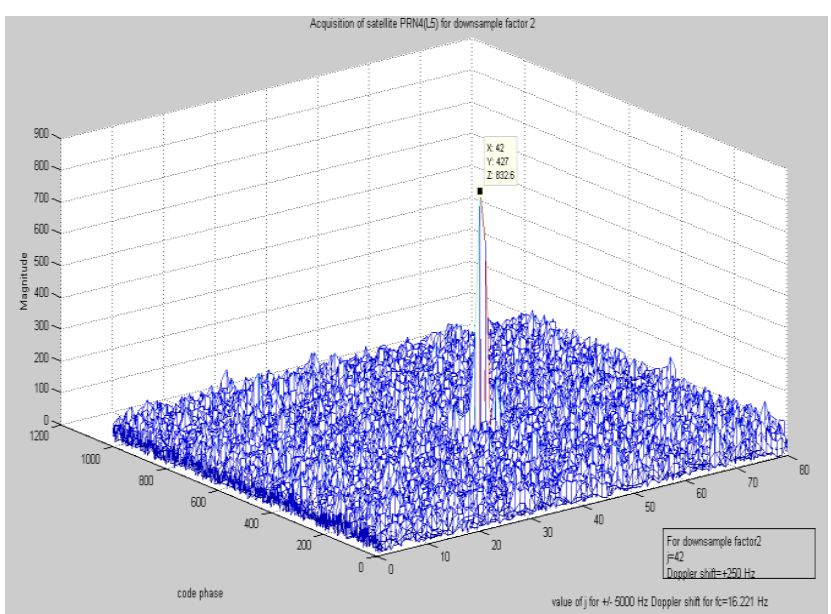

Fig.12 Acquisition results of satellite 4 for down sample factor 3 


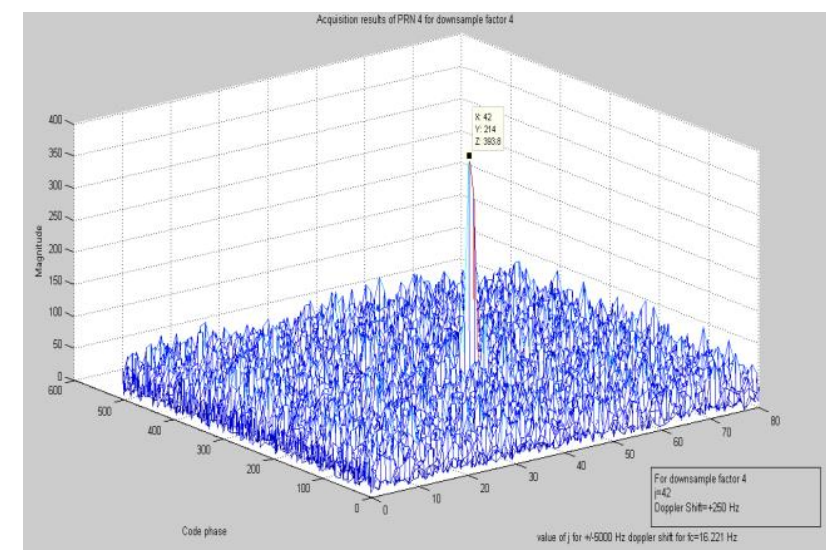

Fig.13 Acquisition results of satellite 4for down sample factor 4

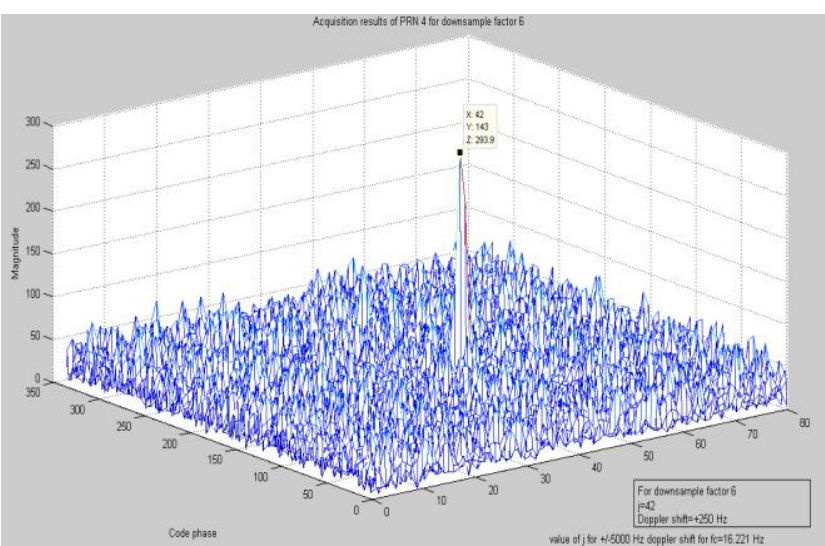

Fig 14 Acquisition results of satellite 4for down sample factor 6

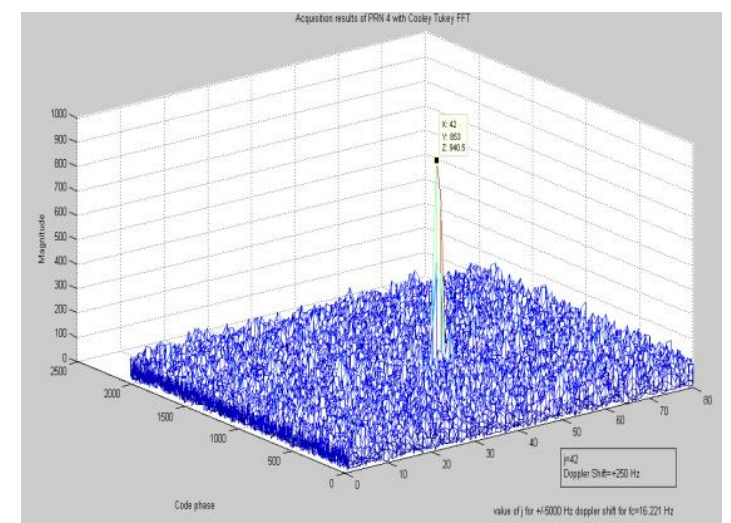

Fig.15 Acquisition results for satellite 4 using Cooley Tukey FFT

Table 1: Acquisition results for different satellites using serial search method

\begin{tabular}{|c|c|c|c|c|}
\hline S.No. & L5-SPS G2 & $\begin{array}{c}\text { Code } \\
\text { phase }\end{array}$ & $\begin{array}{c}\text { Doppler } \\
\text { shift(Hz) }\end{array}$ & $\begin{array}{c}\text { Carrier frequency } \\
\text { (Hz) }\end{array}$ \\
\hline 1 & 1110010111 & 55220 & -1500 & 16219500 \\
\hline 2 & 0110010000 & 30766 & +250 & 16221500 \\
\hline 3 & 0010110001 & 54927 & -125 & 16221500 \\
\hline 4 & 0100111010 & 23290 & +250 & 16221500 \\
\hline 5 & 0000110111 & 39820 & -125 & 16221500 \\
\hline 6 & 1101011000 & 2270 & -125 & 16221500 \\
\hline 7 & 0010100000 & 29315 & +375 & 16221500 \\
\hline
\end{tabular}

Table 2: Acquisition results for different satellites using Parallel Code Phase Search Acquisition Algorithm

\begin{tabular}{|c|c|c|c|c|}
\hline S.No. & L5-SPS G2 & $\begin{array}{c}\text { Code phase in } \\
\mathbf{8 1 8 4} \text { samples }\end{array}$ & $\begin{array}{c}\text { Code phase in } \\
\mathbf{5 6 0 0 0} * \text { 2 samples }\end{array}$ & $\begin{array}{c}\text { Doppler } \\
\text { shift(Hz) }\end{array}$ \\
\hline 1 & 1110010111 & 2020 & 56533 & -2125 \\
\hline 2 & 0110010000 & 1126 & 31501 & +250 \\
\hline 3 & 0010110001 & 2009 & 56225 & -125 \\
\hline 4 & 0100111010 & 853 & 23857 & +250 \\
\hline 5 & 0000110111 & 1457 & 40769 & -250 \\
\hline 6 & 1101011000 & 85 & 2353 & -125 \\
\hline 7 & 0010100000 & 1073 & 30017 & -125 \\
\hline
\end{tabular}


Performance Analysis of Acquisition Algorithms for Navic

Table 3: Acquisition Results of satellite 4 using sub sampled FFT

\begin{tabular}{|c|c|c|c|c|c|}
\hline S.No. & $\mathbf{d}$ & Code phase & $\begin{array}{c}\text { Carrier } \\
\text { frequency(Hz) }\end{array}$ & $\begin{array}{c}\text { time } \\
\text { (sec) }\end{array}$ & $\mathbf{d}^{*} \log (\mathbf{d})$ \\
\hline 1 & - & 853 & 16221250 & 17.1007 & \\
\hline 2 & 2 & $426 * 2+1=853$ & 16221250 & 16.4522 & 2 \\
\hline 3 & 3 & $284 * 3+1=853$ & 16221250 & 16.3571 & 4.75 \\
\hline 4 & 4 & $213 * 4+1=853$ & 16221250 & 16.2946 & 8 \\
\hline 5 & 6 & $142 * 6+1=853$ & 16221250 & 15.878274 & 15.51 \\
\hline
\end{tabular}

Table 4: Acquisition results for different satellites using Cooley Tukey FFT method

\begin{tabular}{|c|c|c|c|c|}
\hline S.No. & L5-SPS G2 & $\begin{array}{c}\text { Code phase in 8184 } \\
\text { samples }\end{array}$ & $\begin{array}{c}\text { Code phase in } \\
\mathbf{5 6 0 0 0 * 2 ~ s a m p l e s ~}\end{array}$ & $\begin{array}{c}\text { Doppler } \\
\text { shift(Hz) }\end{array}$ \\
\hline 1 & 1110010111 & 2020 & 56533 & -2125 \\
\hline 2 & 0110010000 & 1126 & 31501 & +250 \\
\hline 3 & 0010110001 & 2009 & 56225 & -125 \\
\hline 4 & 0100111010 & 853 & 23857 & +250 \\
\hline 5 & 0000110111 & 1457 & 40769 & -250 \\
\hline 6 & 1101011000 & 85 & 2353 & -125 \\
\hline 7 & 0010100000 & 1073 & 30017 & -125 \\
\hline
\end{tabular}

Table 5: Comparison of acquisition results for satellite 4 using different acquisition algorithms

\begin{tabular}{|c|c|c|c|}
\hline Acquisition Algorithm & Code phase & $\begin{array}{c}\text { Carrier } \\
\text { frequency(MHz) }\end{array}$ & Time (sec) \\
\hline Serial search & 23290 & 16.221500 & 1079.7422 \\
\hline $\begin{array}{c}\text { Parallel Code Phase Search } \\
\text { Acquisition Algorithm }\end{array}$ & 23857 & 16.221250 & 17.7001 \\
\hline $\begin{array}{c}\text { FFT Acquisition Algorithm } \\
\text { using Cooley Tukey FFT }\end{array}$ & 23857 & 16.221250 & 119.01 \\
\hline
\end{tabular}

\section{CONCLUSION}

In this paper, various acquisition algorithms for NavIC signals are simulated and analyzed. It is observed that, Parallel FFT(17.7001 s) is faster than Serial Search acquisition algorithm(1079.7422 s).Reduction in the acquisition time and computation complexity is observed by the exploitation of properties of sparse FFT, Fourier transform and decimator and by the use of sub sampled FFT. From the simulation, it is observed that the acquisition time is decreased from (17.7001 s) to (15.878274 s) for down sampling factor 6 without change in the code phase and Doppler frequency shift. The Cooley-Tukey algorithm is also analyzed, it is noticed that, in comparison with Serial search Acquisition algorithm (1079.7422 s),Parallel Code Phase Search(17.7001 s) and Cooley Tukey FFT(119.01 s) Acquisition algorithm is faster.

\section{REFERENCES}

1. Hofmann-Wellenhof, B., Lichtenegger, H., and Collins, J., "Globa Positioning System: Theory and Practice," 5th edition, Springer-Verlog, Berlin Heidelberg New York, 389 pp. 2001.

2. Parkinson, B.W., "Global Positioning System: Theory and Applications", Vol. I, AIAA Publication, 1996

3. Jitender Singh,Sc 'D' and J. Rammohan, Sc'G' 'Indian Regional Navigation Satellite System and its Defence Applications",NAVCOM-2012 December, Hyderabad.

4. Bhaskaranarayana, A. (July 15th 2008) Indian IRNSS \& GAGAN, Presentation to COSPAR Meeting, Montreal.

5. Kaplan, E.D., "Understanding GPS: Principle and Applications," 2nd Edition, Artech HousePublishers, 2005

6. Misra. P and Enge. P., "Global Positioning System: Signals, Measurements, and Performance," Ganga-Jamuna Press, 2001

7. S. Naveen Pitchumani, S. ArunSundar, T. Srinivasan, and S. Savithri," Mathematical Modelling of Indian Regional Navigation Satellite System Receiver”, Defence Science Journal, Vol. 67, No. 4, July 2017

8. Pooja V.Thakar, Hiren Mewada "Receiver Acquisition Algorithms and their Comparisons for BOC modulated Satellite Navigation Signal", 2012 International Conference on Communication Systems and Network Technologies.

9. M.Venu Gopala Rao and D. Venkata Ratnam," Faster Acquisition Technique for Software-defined GPS Receivers",Defence Science Journal, Vol. 65, No. 1, January 2015

10. D.Venkata Ratnam, Ashish Pasha, Swathi P, M.Venu Gopala Rao," Acquisition of GPS L1 Signals Using Cooley-Tukey FFT Algorithm”, Signal Processing, Computing and Control(ISPCC),2013 IEEE Conference.

11. IRNSS Signal In Space ICD for standard positioning service version 1.1, August 2017 (http://www.isro.gov.in/irnss-programme).

12. Anil Manandhar "FPGA-based Tracking System for GNSS Receivers", June 6, 2017.

13. Fredrik Johansson, Rahman Mollaei, Jonas Thor, Jorgen Uusitalo, "GPS Satellite Signal Acquisition and Tracking" August 21,1998

14. Rishija Misra and Shubham Palod,"Code and Carrier Tracking Loops for GPS C/A Code",International Journal of Pure and Applied Sciences and Technology,2011

15. James Bao-Yen Tsui "Fundamentals of Global Positioning System Receivers: A Software Approach" Copyright 2000 John Wiley \& Sons, Inc.

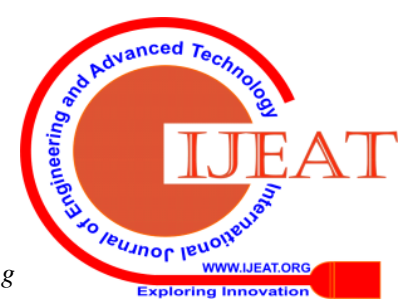




\section{AUTHOR PROFILE}

Author Dr. N. Alivelu Manga, completed her B. Tech from VNRVJIET in 2001 and received M. Tech from JNTUH in 2008. She obtained Ph. D from JNTUH in 2017. Currently working as Associate. Professor in the Dept of ECE CBIT. She published 7 international journals and 10 international conference papers. Having 18 years of experience in teaching and her research interest includes VLSI System Design, GNSS/ NavIC Applications. 